\title{
Finite-Time Stability and Stabilization of Nonlinear Quadratic Systems with Jumps
}

\author{
Minsong Zhang \\ School of Mathematics and Computer Science, Hubei University of Arts and Science, Xiangyang, Hubei 441053, China \\ Correspondence should be addressed to Minsong Zhang; minsongzhang0313@163.com
}

Received 9 September 2013; Revised 5 January 2014; Accepted 15 January 2014; Published 27 February 2014

Academic Editor: Vu Phat

Copyright (C) 2014 Minsong Zhang. This is an open access article distributed under the Creative Commons Attribution License, which permits unrestricted use, distribution, and reproduction in any medium, provided the original work is properly cited.

\begin{abstract}
This paper investigates the problems of finite-time stability and finite-time stabilization for nonlinear quadratic systems with jumps. The jump time sequences here are assumed to satisfy some given constraints. Based on Lyapunov function and a particular presentation of the quadratic terms, sufficient conditions for finite-time stability and finite-time stabilization are developed to a set containing bilinear matrix inequalities (BLIMs) and linear matrix inequalities (LMIs). Numerical examples are given to illustrate the effectiveness of the proposed methodology.
\end{abstract}

\section{Introduction}

Most practical systems, such as missile systems and satellite systems, possess a typical characterization that their operating times always have a finite duration. In this case, the main concern for the researchers is the stability over a fixed finitetime interval rather than the classical Lyapunov asymptotic stability, although the Lyapunov theory is pervasive in control fields from linear methods to nonlinear systems. Usually, a system is finite-time stability (FTS), if, given a finite duration at first, its state is contained within some prescribed bound during this finite duration. The problem of finite-time stability analysis for the control plants has been extensively investigated and a great number of results on this topic have been reported, for example, see [1-6] and the references therein.

At the same time, there are a number of real evolution processes in which the states are subjected to rapid changes at certain time instants. It has been shown that the finite-time stability of a continuous dynamical system could be destroyed by such rapid changes. Therefore, it is important to study the jump's influence on FTS of the control systems. There are several research works that appeared in the literature on control systems with jumps. In [7], the problem of finite-time stabilization for linear systems via jump control is researched through Lyapunov functions. In [8], FTS for time-varying linear systems with jumps is discussed, where a necessary and sufficient condition was obtained. Note that this condition is difficult to test. Considering this, a sufficient condition involving two coupled differential-difference linear matrix inequalities for FTS is presented in [8], which is much easier to be handled.

Recently, the issue of analysis and design of nonlinear quadratic systems has received increasing interest. The Lyapunov asymptotic stability of quadratic systems has achieved great success both in theory and in practice, for example, see [9-15]. The FTS problem for quadratic systems is considered and corresponding sufficient conditions via state feedback controller are derived in terms of LMIs in [15]. However, FTS analysis of nonlinear quadratic systems with jumps has not been fully investigated.

In this paper, we are interested in FTS and finite-time stabilization for nonlinear quadratic systems with jumps. Based on the Lyapunov function and a particular presentation of the quadratic terms, sufficient conditions for FTS and finite-time stabilization are presented for such quadratic systems in terms of LMIs. Furthermore, two examples are presented to illustrate its effectiveness.

Throughout this paper, standard notation is adopted. Given a scalar $\alpha>0,[\alpha]$ denotes the maximum integer which is less than or equal to $\alpha$. The class of jump time sequences satisfying $\inf _{k}\left\{t_{k}-t_{k-1}\right\} \geq \beta$ is denoted by $\gamma_{\text {min }}(\beta)$. 
For real symmetric matrices $X$ and $Y$, the notation $X \geq$ $Y$ (respectively, $X>Y$ ) means that the matrix $X-Y$ is positive semidefinite (respectively, positive definite). $A \otimes B$ is the Kronecker product of the pair of $(A, B)$. Matrices, if not explicitly stated, are assumed to have compatible dimensions.

\section{Problem Statement}

Consider a class of nonlinear quadratic systems with finite jumps described as

$$
\begin{gathered}
\dot{x}(t)=A_{c} x(t)+B_{c}(x(t))+F_{c} u_{c}(t), \quad t \neq t_{k}, \\
\Delta x(t)=\left(A_{d}-I\right) x\left(t^{-}\right)+B_{d}\left(x\left(t^{-}\right)\right)+F_{d} u_{d}\left(t^{-}\right), \\
t=t_{k}, \\
x\left(t_{0}\right)=x_{0}, \quad t_{0}=0,
\end{gathered}
$$

where $x(t) \in R^{n}, u_{c}(t) \in R^{m_{1}}$, and $u_{d}(t) \in R^{m_{2}}$ are system state, continuous control input, and jump control input, respectively. $\Delta x\left(t_{k}\right)=x\left(t_{k}^{+}\right)-x\left(t_{k}^{-}\right)$, where $x\left(t_{k}\right)=$ $x\left(t_{k}^{+}\right)=\lim _{h \rightarrow 0^{+}} x\left(t_{k}+h\right), x\left(t_{k}^{-}\right)=\lim _{h \rightarrow 0^{+}} x\left(t_{k}-h\right)$ and $0<t_{1}<t_{2}<\cdots t_{r} \leq T<t_{r+1}<\cdots, \lim _{k \rightarrow \infty} t_{k}=\infty$ with $T$ as a positive scalar. Denote

$$
\begin{gathered}
B_{c}(x(t))=\left[\begin{array}{c}
x^{T}(t) B_{c 1} x(t) \\
x^{T}(t) B_{c 2} x(t) \\
\vdots \\
x(t)^{T} B_{c n} x(t)
\end{array}\right], \\
B_{d}\left(x\left(t^{-}\right)\right)=\left[\begin{array}{c}
x^{T}\left(t^{-}\right) B_{d 1} x\left(t^{-}\right) \\
x^{T}\left(t^{-}\right) B_{d 2} x\left(t^{-}\right) \\
\vdots \\
x^{T}\left(t^{-}\right) B_{d n} x\left(t^{-}\right)
\end{array}\right],
\end{gathered}
$$

where $B_{c i}, B_{d i} \in R^{n \times n}, i=1, \ldots, n$.

Let us define matrices $B_{c q}, B_{d q} \in R^{n \times n^{2}}$ as following

$$
\begin{aligned}
B_{c q} & =\left[\begin{array}{cccc}
B_{c 1(1)} & B_{c 1(2)} & \cdots & B_{c 1(n)} \\
\vdots & \vdots & \ddots & \vdots \\
B_{c n(1)} & B_{c n(2)} & \cdots & E_{c n(n)}
\end{array}\right], \\
B_{d q} & =\left[\begin{array}{cccc}
B_{d 1(1)} & B_{d 1(2)} & \cdots & B_{d 1(n)} \\
\vdots & \vdots & \ddots & \vdots \\
B_{d n(1)} & B_{d n(2)} & \cdots & E_{d n(n)}
\end{array}\right],
\end{aligned}
$$

where $B_{c i(j)}$ and $B_{d i(j)}$ denote the $j$ th row of matrices $B_{c i}$ and $B_{d i}$, respectively. Then, the system (1) can be read as follows:

$$
\begin{array}{rl}
\dot{x}(t) & =\left(A_{c}+B_{c q}\left(x(t) \otimes I_{n}\right)\right) x(t)+F_{c} u_{c}(t), \quad t \neq t_{k}, \\
\Delta x(t) & =\left(A_{d}-I+B_{d q}\left(x\left(t^{-}\right) \otimes I_{n}\right)\right) x\left(t^{-}\right)+F_{d} u_{d}\left(t^{-}\right), \\
t & t=t_{k}, \\
x\left(t_{0}\right) & =x_{0}, \quad t_{0}=0 .
\end{array}
$$

In what follows, we introduce two lemmas, which are essential for the developments in the next section.

Lemma 1 (see [14]). For any matrices $A, P \in R^{n \times n}, D \in R^{n \times n_{f}}$, $E, N \in R^{n_{f} \times n}$, and $F \in R^{n_{f} \times n_{f}}$, where $P>0,\|F\| \leq 1$, and $a$ scalar $\varepsilon>0$, the following inequalities hold

(1) $D F N+N^{T} F^{T} D^{T} \leq \varepsilon^{-1} D D^{T}+\varepsilon N^{T} N$,

(2) if $P-\varepsilon D D^{T}>0$, then

$(A+D F E)^{T} P^{-1}(A+D F E) \leq A^{T}\left(P-\varepsilon D D^{T}\right)^{-1} A+\varepsilon^{-1} E^{T} E$.

Lemma 2 (see [12]). Consider matrix $P \in R^{n \times n}$ with $P>0$ and a vector $v$ such that $\|\nu\|=1$. Every point on the boundary of an ellipsoid, $\partial \mathscr{E}(P)=\left\{x \in R^{n}: x^{T} P x=1\right\}$, can be parameterized by $x=P^{-1 / 2} Q v$, where $Q^{T} Q=1$.

\section{Main Results}

In this section, we establish sufficient conditions of FTS and finite-time stabilization for the nonlinear quadratic system (1) based on Lyapunov functions and a particular presentation for the quadratic terms.

First, we introduce the definition of FTS for nonlinear quadratic system (1).

Definition 3 (see [3]). Given a scalar $T>0$ and matrices $R>$ $0, S>0$ with $S<R$; system (1) with $u_{c}(t)=0$ and $u_{d}(t)=0$ is said to be FTS with respect to $(T, R, S)$, if

$$
x_{0}^{T} R x_{0} \leq 1 \Longrightarrow x(t)^{T} S x(t)<1, \quad \forall t \in[0, T] .
$$

Now, we provide a sufficient condition for FTS of the system (1) with $u_{c}(t)=0$ and $u_{d}(t)=0$.

Theorem 4. For a prescribed scalar $\beta$ satisfying $0<\beta \leq T$, assume that there exist scalars $\mu \geq 1, \varepsilon_{i}>0$, and $i=1,2$ and $a$ matrix $P>0$ such that

$$
\begin{aligned}
& {\left[\begin{array}{cc}
A_{c}^{T} P+P A_{c}+\varepsilon_{1} I+\frac{\ln \mu}{\beta} P & P B_{c q} \\
* & -\varepsilon_{1}\left(S \otimes I_{n}\right)
\end{array}\right]<0,} \\
& {\left[\begin{array}{ccc}
-\mu P+\varepsilon_{2} I & A_{d}^{T} P & 0 \\
* & -P & P B_{d q} \\
* & * & -\varepsilon_{2}\left(S \otimes I_{n}\right)
\end{array}\right] \leq 0,} \\
& S \leq P, \\
& P<R \text {, }
\end{aligned}
$$

then when $\mu>1$, system (1) with $u_{c}(t)=0$ and $u_{d}(t)=0$ is FTS with respect to $(T, R, S)$ over $\gamma_{\min }(\beta)$; when $\mu=1$, system (1) with $u_{c}(t)=0$ and $u_{d}(t)=0$ is FTS with respect to $(T, R, S)$ for any jump time sequences $\left\{t_{k}\right\}, k \in[1, r]$.

Proof. Note (7) and from the Schur's complement, we obtain

$$
A_{c}^{T} P+P A_{c}+\varepsilon_{1} I+\frac{\ln \mu}{\beta} P+\varepsilon_{1}^{-1} P B_{c q}\left(S^{-1} \otimes I_{n}\right) B_{c q}^{T} P<0 .
$$


Before and after multiplying (8), respectively, by $\operatorname{diag}\{I, P^{-1}, \underbrace{I, \cdots, I}_{n}\}$ and then applying the Schur complement, we get

$$
-\mu P+\varepsilon_{2} I+A_{d}^{T}\left(P^{-1}-\varepsilon_{2}^{-1} B_{d q}\left(S^{-1} \otimes I_{n}\right) B_{d q}^{T}\right)^{-1} A_{d}<0 .
$$

In order to complete the proof, we will first show that the nonlinear quadratic system (1) is FTS with respect to $\left(t_{1}, R, S\right)$. Indeed, when $t \in\left[t_{0}, t_{1}\right)$, we can choose the quadratic Lyapunov function as $V(x(t))=x^{T}(t) P x(t)$ for system (1), where $u_{c}(t)=0$ and $u_{d}(t)=0$. Then, we obtain

$$
\begin{aligned}
\dot{V}(x(t)) & +\frac{\ln \mu}{\beta} V(x(t)) \\
=x^{T}(t)\left(A_{c}^{T} P\right. & +P A_{c}+P B_{c q}\left(x(t) \otimes I_{n}\right) \\
& \left.+\left(x^{T}(t) \otimes I_{n}\right) B_{c q}^{T} P\right) x(t) .
\end{aligned}
$$

By Proposition 1 in [12], it follows that $\dot{V}(x(t))+$ $((\ln \mu) / \beta) V(x(t))$ is negative definite in $\mathscr{E}(S)$, if it is negative definite in $\partial \mathscr{E}(S)$. By Lemma 2 , for $x \in \partial \mathscr{E}(S)$, we can get

$$
\begin{aligned}
\dot{V}(x(t))+\frac{\ln \mu}{\beta} V(x(t)) & \\
=x^{T}(t)\left(A_{c}^{T} P+P A_{c}\right. & \\
& +P B_{c q}\left(S^{-1 / 2} \otimes I_{n}\right)\left(Q \otimes I_{n}\right)\left(v \otimes I_{n}\right) \\
& +\left(v^{T} \otimes I_{n}\right)\left(Q^{T} \otimes I_{n}\right)\left(\left(S^{-1 / 2}\right)^{T} \otimes I_{n}\right) B_{c q}^{T} P \\
& \left.+\frac{\ln \mu}{\beta} P\right) x(t),
\end{aligned}
$$

where $Q^{T} Q=1,\|v\|^{2}=1$. From Lemma 1, substituting (11) to the above inequality, for any $\varepsilon_{1}>0$ and $x \in \partial \mathscr{E}(S)$, yields to

$$
\begin{gathered}
\dot{V}(x(t))+\frac{\ln \mu}{\beta} V(x(t)) \\
\leq x^{T}(t)\left(A_{c}^{T} P+P A_{c}+\varepsilon_{1} I+\varepsilon^{-1} P B_{c q}\left(I_{n} \otimes S^{-1}\right) B_{c q}^{T}\right. \\
\left.+\frac{\ln \mu}{\beta} P\right) x(t)
\end{gathered}
$$

$<0$.

Thus, for all $x \in \mathscr{E}(S)$, it is easy to see that

$$
\dot{V}(x(t))<-\frac{\ln \mu}{\beta} V(x(t))<0, \quad t \in\left[0, t_{1}\right) .
$$

Divide both sides of (16) by $V(x)$, and integrate from 0 to $t$ with $t \in\left[0, t_{1}\right)$, it follows that

$$
V(x(t))<e^{-((\ln \mu) / \beta) t} V(x(0)) .
$$

Therefore, (9) and (10) give that, for all $t \in\left[0, t_{1}\right)$

$$
\begin{aligned}
x^{T}(t) S x(t) & \leq x^{T}(t) P x(t) \\
& <e^{-((\ln \mu) / t) \beta} V(x(0)) \\
& <x^{T}(0) R x(0) \\
& \leq 1
\end{aligned}
$$

which shows that any trajectory starting from the set $S$ cannot exit in $\mathscr{E}(S)$ for $t \in\left[0, t_{1}\right)$.

When $t=t_{1}$,

$$
\begin{aligned}
V\left(x\left(t_{1}^{-}\right)\right)= & x^{T}\left(t_{1}^{-}\right)\left(A_{d}+B_{d q}\left(x\left(t_{1}^{-}\right) \otimes I_{n}\right)\right)^{T} \\
& \times P\left(A_{d}+B_{d q}\left(x\left(t_{1}^{-}\right) \otimes I_{n}\right)\right) x\left(t_{1}^{-}\right) .
\end{aligned}
$$

Lemmas 1 and 2 yield to

$$
\begin{aligned}
& V\left(x\left(t_{1}^{-}\right)\right) \\
& \quad \leq x\left(t_{1}^{-}\right) A_{d}^{T}\left(P^{-1}-\varepsilon_{2}^{-1} B_{d q}\left(S^{-1} \otimes I_{n}\right) B_{d q}^{T}\right)^{-1} A_{d} x\left(t_{1}^{-}\right),
\end{aligned}
$$

where $\varepsilon_{2}>0$. Substituting (12) into the above inequality, then

$$
V\left(x\left(t_{1}^{-}\right)\right) \leq \mu V\left(x\left(t_{1}^{-}\right)\right) .
$$

Therefore, it follows from (17) and (21) that

$$
V\left(x\left(t_{1}^{-}\right)\right)<\mu e^{-((\ln \mu) / \beta) t_{1}} V(x(0)) .
$$

Since $t_{k}-t_{k-1} \geq \beta$ implies that $t_{1} \geq \beta$, it is easy to see that

$$
V\left(x\left(t_{1}^{-}\right)\right)<V(x(0)) .
$$

Thus, using (9) and (10), we have

$$
\begin{aligned}
x^{T}\left(t_{1}^{-}\right) S x\left(t_{1}^{-}\right) & \leq V\left(x\left(t_{1}^{-}\right)\right) \\
& <V(x(0)) \\
& <x^{T}(0) R x(0) \\
& \leq 1 .
\end{aligned}
$$

Thus, (18) and (24) imply that the nonlinear quadratic system (1) is FTS with respect to $\left(t_{1}, R, S\right)$. Based on mathematical induction, one can easily verify that the nonlinear quadratic system (1) is FTS with respect to $\left(t_{k}, R, S\right), k \in[2, r]$. Note that $V(x(t))$ is strictly decreasing along the trajectories of quadratic system (1) for $t \in\left[t_{r}, T\right]$; we have

$$
x^{T}(T) S x(T) \leq V(x(T))<V\left(x\left(t_{r}\right)\right) \leq 1 .
$$

The proof is complete. 
Remark 5. When $\mu>1$, the jumps may destroy FTS of system (1), so it should not occur frequently. In this case, $\inf _{k}\left\{t_{k}-\right.$ $\left.t_{k-1}\right\} \geq \beta$ implies that the jump time $r$ is satisfying $r \leq[T / \beta]$.

Remark 6. When $\mu=1$, since the conditions in Theorem 4 are not related to $\beta$, the quadratic system with jumps is FTS regardless of how often or how seldom jumps occur during the time interval $[0, T]$.

Remark 7. Compared with the method presented in [8], the quadratic Lyapunov function used in this paper is not required to be strictly decreasing along the trajectories of the nonlinear quadratic system (1).

Now, we extend Theorem 4 to the design context. Consider the following nonlinear control law:

$$
\begin{gathered}
u_{c}(t)=K_{c} x(t)+\left[\begin{array}{c}
x^{T}(t) K_{c q 1} x(t) \\
x^{T}(t) K_{c q 2} x(t) \\
\vdots \\
x^{T}(t) K_{c q m_{1}} x(t)
\end{array}\right], \\
u_{d}\left(t^{-}\right)=K_{d} x\left(t^{-}\right)+\left[\begin{array}{c}
x^{T}\left(t^{-}\right) K_{d q 1} x\left(t^{-}\right) \\
x^{T}\left(t^{-}\right) K_{d q 2} x\left(t^{-}\right) \\
\vdots \\
x^{T}\left(t^{-}\right) K_{d q m_{2}} x\left(t^{-}\right)
\end{array}\right],
\end{gathered}
$$

where $K_{c} \in R^{m_{1} \times n}, K_{d} \in R^{m_{2} \times n}, K_{c q i} \in R^{n \times m_{1}}, i=1, \ldots, m_{1}$, and $K_{d q j} \in R^{n \times m_{2}}, j=1, \ldots, m_{2}$. Based on (26), we define the matrices $K_{c q} \in R^{m_{1} \times n^{2}}$ and $K_{d q} \in R^{m_{2} \times n^{2}}$ as follows:

$$
\begin{gathered}
K_{c q}=\left[\begin{array}{cccc}
K_{c q 1(1)} & K_{c q 1(2)} & \ldots & K_{c q 1(n)} \\
\vdots & \vdots & \ddots & \vdots \\
K_{c q m_{1}(1)} & K_{c q m_{1}(2)} & \ldots & K_{c q m_{1}(n)}
\end{array}\right], \\
K_{d q}=\left[\begin{array}{cccc}
K_{d q 1(1)} & K_{c q 1(2)} & \ldots & K_{d q 1(n)} \\
\vdots & \vdots & \ddots & \vdots \\
K_{d q m_{2}(1)} & K_{c q m_{2}(2)} & \ldots & K_{c q m_{2}(n)}
\end{array}\right],
\end{gathered}
$$

then the corresponding continuous control law and jump control law are, respectively,

$$
\begin{gathered}
u_{c}(t)=\left(K_{c}+K_{c q}\left(x(t) \otimes I_{n}\right)\right) x(t), \\
u_{d}(t)=\left(K_{d}+K_{d q}\left(x\left(t^{-}\right) \otimes I_{n}\right)\right) x\left(t^{-}\right) .
\end{gathered}
$$

Then, the corresponding closed-loop system is defined by

$$
\begin{aligned}
& \dot{x}(t)= {\left[\left(A_{c}+F_{c} K_{c}\right)\right.} \\
&\left.+\left(B_{c q}+F_{c} K_{c q}\right)\left(x(t) \otimes I_{n}\right)\right] x(t), \quad t \neq t_{k}, \\
& \Delta x(t)= {\left[\left(A_{d}-I+F_{d} K_{d}\right)\right.} \\
&\left.+\left(B_{d q}+F_{d} K_{d q}\right)\left(x\left(t^{-}\right) \otimes I_{n}\right)\right] x\left(t^{-}\right), \quad t=t_{k}, \\
& x\left(t_{0}\right)=x_{0}, \quad t_{0}=0 .
\end{aligned}
$$

The following theorem presents a sufficient condition of finite-time stabilization for the closed-loop system (29).

Theorem 8. For a prescribed scalar $\beta>0$, assume that there exist positive scalars $\mu \geq 1, \lambda_{i}, i=1,2$ and matrices $X>0 \epsilon$ $R^{n \times n}, L_{c} \in R^{m_{1} \times n}, L_{c q} \in R^{m_{1} \times n^{2}}, L_{d} \in R^{m_{2} \times n}$, and $L_{d q} \in R^{m_{2} \times n^{2}}$ such that

$$
\begin{aligned}
& {\left[\begin{array}{ccc}
\Psi & \lambda_{1} B_{c q}+F_{c} L_{c q} & X \\
* & -\lambda_{1}\left(S \otimes I_{n}\right) & 0 \\
* & * & -\lambda_{1} I
\end{array}\right]<0} \\
& {\left[\begin{array}{cccc}
-\mu X & X A_{d}^{T}+L_{d}^{T} F_{d}^{T} & 0 & X \\
* & -X & \lambda_{2} B_{d q}+F_{d} L_{d q} & 0 \\
* & * & -\lambda_{2}\left(S \otimes I_{n}\right) & 0 \\
* & * & * & -\lambda_{2} I
\end{array}\right] \leq 0,} \\
& {\left[\begin{array}{cc}
-X & I \\
* & -S
\end{array}\right] \leq 0,} \\
& {\left[\begin{array}{cc}
-R & I \\
* & -X
\end{array}\right]<0,}
\end{aligned}
$$

with

$$
\Psi=X A_{c}^{T}+A_{c} X+F_{c} L_{c}+L_{c}^{T} F_{c}^{T}+\frac{\ln \mu}{\beta} X .
$$

Then

(1) if $\mu>1$, the closed-loop system (29) is finite-time stabilizable with respect to $(T, R, S)$ over $\gamma_{\min }(\beta)$ and the stabilizing state feedback controller (28) is given by $K_{c}=L_{c} X^{-1}, K_{c q}=L_{c q} / \lambda_{1}, K_{d}=L_{d} X^{-1}$, and $K_{d q}=L_{d q} / \lambda_{2}$

(2) if $\mu=1$, the system (29) is finite-time stabilizable with respect to $(T, R, S)$ for any jump time sequences $\left\{t_{k}\right\}$, $k \in[1, r]$ and the stabilizing state feedback controller (28) is given by $K_{c}=L_{c} X^{-1}, K_{c q}=L_{c q} / \lambda_{1}, K_{d}=$ $L_{d} X^{-1}$, and $K_{d q}=L_{d q} / \lambda_{2}$.

Proof. Since the proof of Theorem 8 is a natural extension from Theorem 4, we omit it.

Remark 9. Due to Remark 8 in [5], the quadratic terms on the presented control law (28) can be explained as a counteraction to the influence of the quadratic terms of the system. Especially, when $m_{1}=m_{2}=n, F_{c}$ and $F_{d}$ are nonsingular matrices; it is possible for the closed system to eliminate the quadratic terms completely through $K_{c q}=$ $-F^{-1} B_{c q}$ and $K_{d q}=-F^{-1} B_{d q}$.

\section{Numerical Examples}

In this section, we will present two examples to illustrate the effectiveness of our method. In Theorem $4, \mu$ and $P$ are variables, then (7) and (8) are not LMIs. Obviously, the primary matter is the solvability of the inequalities (7)-(10). For eliminating the coupling, we directly fix $\mu$ with a constant; then (7) and (8) are LMIs and can solved through MatLab. For Theorem 8 , we have the similar process. 
Example 10. Consider the system (1) with the following parameters:

$$
\begin{aligned}
& A_{c}=\left[\begin{array}{ll}
2 & 1 \\
0 & 1
\end{array}\right], \quad B_{c q}=\left[\begin{array}{llll}
0 & 0 & -0.5 & 0 \\
0 & 0 & -0.5 & 0
\end{array}\right], \\
& F_{c}=\left[\begin{array}{l}
1 \\
1
\end{array}\right], \quad A_{d}=\left[\begin{array}{cc}
1.1 & 0.02 \\
0 & 1.1
\end{array}\right] \text {, } \\
& B_{d q}=\left[\begin{array}{cccc}
0.6 & 0 & 0.3 & 0 \\
0 & 0 & 0 & 0
\end{array}\right], \quad F_{d}=\left[\begin{array}{l}
1 \\
0
\end{array}\right], \quad \beta=0.5 \text {. }
\end{aligned}
$$

Our aim is to solve the finite-time stabilization problem by using nonlinear state feedback with respect to $(R, S, T)$, where

$$
R=\left[\begin{array}{ll}
1 & 0 \\
0 & 1
\end{array}\right], \quad S=\left[\begin{array}{cc}
0.1 & 0 \\
0 & 0.1
\end{array}\right], \quad T=2.3 .
$$

It is noticed that the jump system may destroy FTS of the closed-loop system.

Through Theorem 8 with $\mu=1.211$, we obtain the continuous state feedback control law $u_{c}(t)=$ $\left(\left[\begin{array}{ll}-5.7815 & -5.7819\end{array}\right]+\left[\begin{array}{llll}0.5 & 0 & 0 & 0\end{array}\right]\left(I_{2} \otimes x(t)\right)\right) x(t)$ and the discrete state feedback control law $u_{d}(t)=$ $\left(\left[\begin{array}{ll}-1.3987 & -0.1241\end{array}\right]+\left[\begin{array}{llll}-0.6 & 0 & -0.3 & 0\end{array}\right]\left(x(t) \otimes I_{2}\right)\right) x(t)$.

Example 11. Consider the system (1) with the following parameters:

$$
\begin{aligned}
& A_{c}=\left[\begin{array}{cc}
0 & 1 \\
1 & -2
\end{array}\right], \quad B_{c q}=\left[\begin{array}{cccc}
0 & 0 & 0.2 & 0 \\
0 & 0 & -0.5 & 0
\end{array}\right], \\
& F_{c}=\left[\begin{array}{l}
0 \\
1
\end{array}\right], \quad A_{d}=\left[\begin{array}{cc}
1.2 & 0.2 \\
0 & 0.5
\end{array}\right] \text {, } \\
& B_{d q}=\left[\begin{array}{cccc}
0.6 & 0 & 0.3 & 0 \\
0 & 0 & 0 & 0
\end{array}\right], \quad F_{d}=\left[\begin{array}{l}
1 \\
0
\end{array}\right] \text {. }
\end{aligned}
$$

It is noticed that the jump system is helpful to FTS of closed-loop system by the discrete state feedback control law. Our goal is to solve the finite-time stabilization problem by using nonlinear state feedback with the same $R, S$, and $T$ given in Example 10.

Based on Theorem 8 and a given $\mu=1$, we obtain the continuous state feedback control law $u_{c}(t)=$ $\left(\left[\begin{array}{ll}-10.7383 & -81.2935\end{array}\right]+\left[\begin{array}{llll}0 & 0 & 0.4865 & 0\end{array}\right]\left(x(t) \otimes I_{n}\right)\right) x(t)$ and the discrete state feedback control law $u_{d}(t)=$ $\left(\left[\begin{array}{ll}-2.2464 & 0.8832\end{array}\right]+\left[\begin{array}{llll}-0.6 & 0 & -0.3 & 0\end{array}\right]\left(x(t) \otimes I_{n}\right)\right) x(t)$.

\section{Conclusion}

In this paper, the problems of FTS and finite-time stabilization for nonlinear quadratic systems with jumps are investigated. Sufficient conditions of FTS and finite-time stabilization based on the quadratic Lyapunov function and a particular presentation for the quadratic terms are established in terms of BLMIs and LMIs. And two examples have been provided to explain the effectiveness of our methodology. In the future, we will continue the study of nonlinear quadratic systems with jumps, such as the systems subject to jumps and input saturation or time delay.

\section{Conflict of Interests}

The authors declare that there is no conflict of interests regarding the publication of this paper.

\section{References}

[1] P. Dorato, "Short time stability in linear time-varying systems," in Proceedings of the IRE International Convention Record, pp. 83-87, New York, NY, USA, 1961.

[2] L. Weiss and E. F. Infante, "Finite time stability under perturbing forces and on product spaces," IEEE Transactions on Automatic Control, vol. 12, no. 1, pp. 54-59, 1967.

[3] F. Amato, M. Ariola, and P. Dorato, "Finite-time control of linear systems subject to parametric uncertainties and disturbances," Automatica, vol. 37, no. 9, pp. 1459-1463, 2001.

[4] A. N. Michel and L. Hou, "Finite-time and practical stability of a class of stochastic dynamical systems," in Proceedings of the 47th IEEE Conference on Decision and Control (CDC '08), pp. 3452-3456, Cancun, Mexico, December 2008.

[5] G. Garcia, S. Tarbouriech, and J. Bernussou, "Finite-time stabilization of linear time-varying continuous systems," IEEE Transactions on Automatic Control, vol. 54, no. 2, pp. 364-369, 2009.

[6] F. Amato, M. Ariola, and C. Cosentino, "Finite-time stability of linear time-varying systems: analysis and controller design," IEEE Transactions on Automatic Control, vol. 55, no. 4, pp. 1003$1008,2010$.

[7] L. Liu and J. Sun, "Finite-time stabilization of linear systems via impulsive control," International Journal of Control, vol. 81, no. 6, pp. 905-909, 2008.

[8] F. Amato, R. Ambrosino, M. Ariola, and C. Cosentino, "Finitetime stability of linear time-varying systems with jumps," Automatica, vol. 45, no. 5, pp. 1354-1358, 2009.

[9] F. Amato, C. Cosentino, and A. Merola, "On the region of asymptotic stability of nonlinear quadratic systems," in Proceedings of the 14th Mediterranean Conference on Control and Automation (MED '06), pp. 1-5, Ancona, Italy, June 2006.

[10] F. Amato, C. Cosentino, and A. Merola, "On the region of attraction of nonlinear quadratic systems," Automatica, vol. 43, no. 12, pp. 2119-2123, 2007.

[11] F. Amato, F. Calabrese, C. Cosentino, and A. Merola, "Stability analysis of nonlinear quadratic systems via polyhedral Lyapunov functions," Automatica, vol. 47, no. 3, pp. 614-617, 2011.

[12] G. Valmórbida, S. Tarbouriech, and G. Garcia, "State feedback design for input-saturating quadratic systems," Automatica, vol. 46, no. 7, pp. 1196-1202, 2010.

[13] D. Coutinho and C. E. de Souza, "Nonlinear state feedback design with a guaranteed stability domain for locally stabilizable unstable quadratic systems," IEEE Transactions on Circuits and Systems, vol. 59, no. 2, pp. 360-370, 2012.

[14] Y. Wang, L. Xie, and C. E. de Souza, "Robust control of a class of uncertain nonlinear systems," Systems \& Control Letters, vol. 19, no. 2, pp. 139-149, 1992.

[15] F. Amato, C. Cosentino, and A. Merola, "Sufficient conditions for finite-time stability and stabilization of nonlinear quadratic systems," IEEE Transactions on Automatic Control, vol. 55, no. 2, pp. 430-434, 2010. 


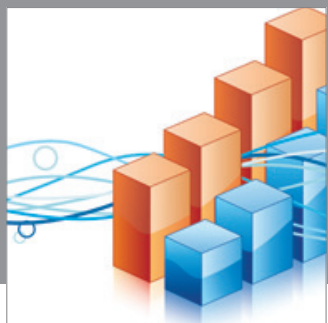

Advances in

Operations Research

mansans

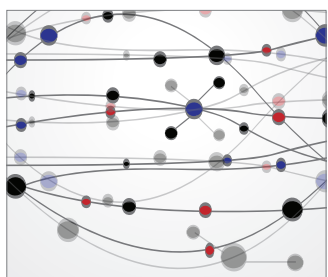

The Scientific World Journal
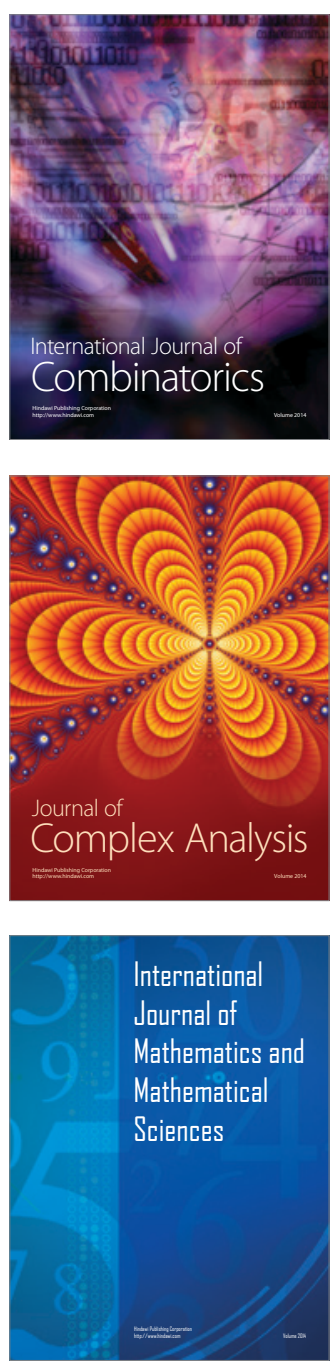
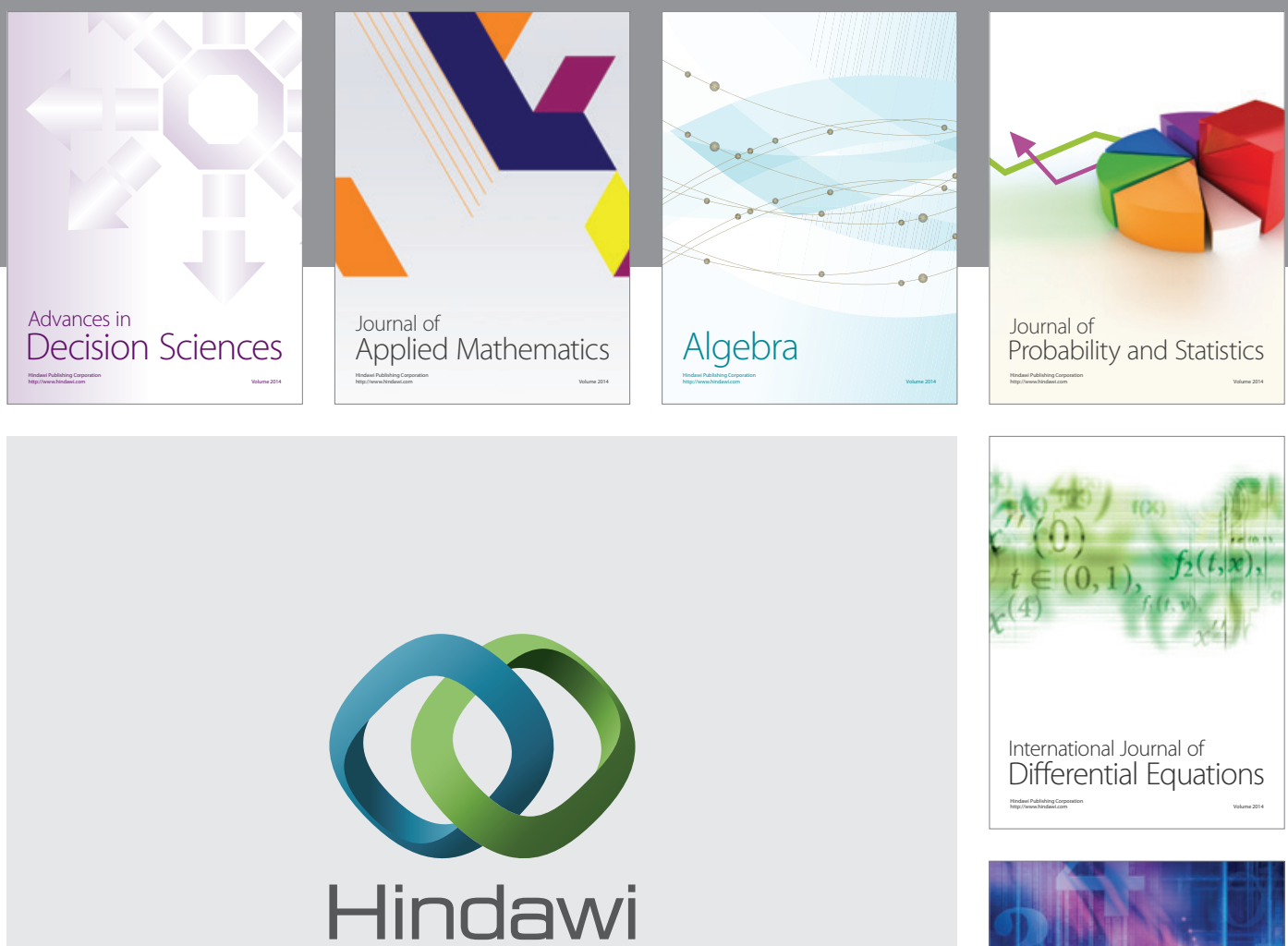

Submit your manuscripts at http://www.hindawi.com
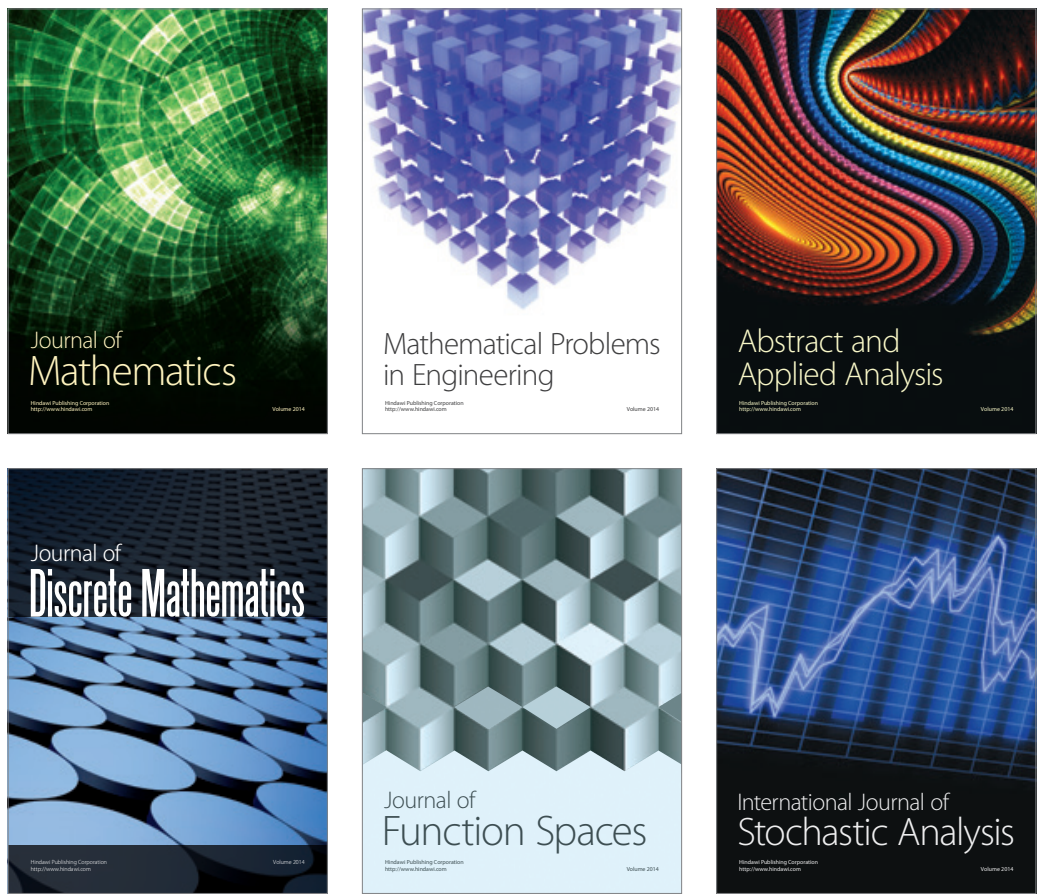

Journal of

Function Spaces

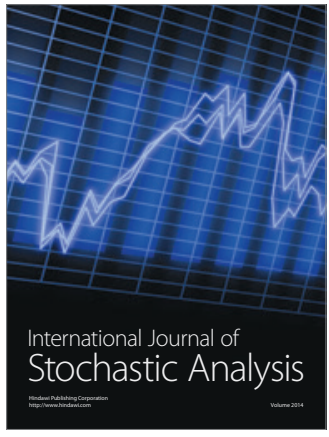

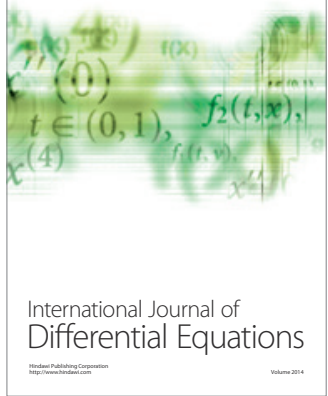
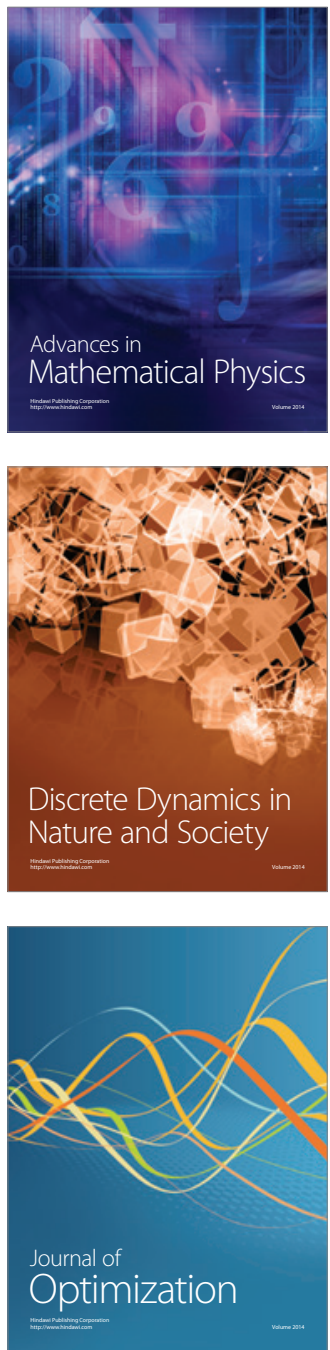